\title{
LOCAL LIMIT APPROXIMATIONS FOR MARKOV POPULATION PROCESSES
}

\author{
SANDA N. SOCOLL* AND \\ A. D. BARBOUR, ${ }^{* * *}$ Universität Zürich
}

\begin{abstract}
In this paper we are concerned with the equilibrium distribution $\Pi_{n}$ of the $n$th element in a sequence of continuous-time density-dependent Markov processes on the integers. Under a $(2+\alpha)$ th moment condition on the jump distributions, we establish a bound of order $O\left(n^{-(\alpha+1) / 2} \sqrt{\log n}\right)$ on the difference between the point probabilities of $\Pi_{n}$ and those of a translated Poisson distribution with the same variance. Except for the factor $\sqrt{\log n}$, the result is as good as could be obtained in the simpler setting of sums of independent, integer-valued random variables. Our arguments are based on the Stein-Chen method and coupling.
\end{abstract}

Keywords: Continuous-time Markov jump process; equilibrium distribution; point probabilities; Stein-Chen method; coupling

2000 Mathematics Subject Classification: Primary 60J75; 62E17

\section{Introduction}

Density-dependent Markov population processes, in which the transition rates depend on the density of individuals in the population, have proved widely useful as models in the social and life sciences; see, for example, the monograph of Kurtz (1981), in which approximations in terms of diffusions are extensively discussed, in the limit as the typical population size $n$ tends to $\infty$. In the present paper we consider local approximation to their equilibrium distributions $\Pi_{n}$. In Socoll and Barbour (2009), a total variation approximation to $\Pi_{n}$ by a suitably translated Poisson distribution was shown to be accurate to order $O\left(n^{-\alpha / 2}\right)$, provided that the jump distributions satisfy a $(2+\alpha)$ th moment condition for some $0<\alpha \leq 1$. Here, we examine the approximation of point probabilities by those of the same translated Poisson distribution, and show in Theorem 1.1 that, under the same assumptions, the error is now of order $O\left(n^{-(\alpha+1) / 2} \sqrt{\log n}\right)$. This is only worse by the logarithmic factor than the best that can be obtained under comparable conditions for sums of independent, integer-valued random variables.

A key ingredient in the proof of the total variation approximation in Socoll and Barbour (2009) was to show that the total variation distance between $\Pi_{n}$ and its unit translate $\Pi_{n} * \delta_{1}$ is of order $O\left(n^{-1 / 2}\right)$. Here, we need to establish a local limit analogue of this theorem. We prove in Section 2 that the differences between the point probabilities of $\Pi_{n}$ and those of its unit translate are uniformly bounded by a quantity of order $O\left(n^{-1} \sqrt{\log n}\right)$. An important step in proving this is to establish that, for some $U \geq 1$, the difference between $\mathrm{P}\left[Z_{n}(t)=k+1 \mid Z_{n}(0)=i\right]$ and $\mathrm{P}\left[Z_{n}(t)=k \mid Z_{n}(0)=i-1\right]$ is of order $O\left(n^{-1} \sqrt{\log n}\right)$, uniformly for $i$ in a set $I$ such

Received 23 February 2009; revision received 9 June 2009.

* Postal address: Angewandte Mathematik, Universität Zürich, Winterthurerstrasse 190, CH-8057 Zürich, Switzerland.

Work supported in part by Schweizerischer Nationalfonds Projekte Nrs 20-107935/1 and 20-117625/1.

** Email address: a.d.barbour@math.uzh.ch 
that $\Pi_{n}\left(I^{\mathrm{c}}\right)=O\left(n^{-1}\right)$. This is achieved by a pathwise comparison of probability densities, and using a martingale concentration inequality. Note that, for sums of independent random variables, the corresponding difference is always 0 , so that this problem does not arise there.

The proof of Theorem 1.1 is undertaken in Section 3. The argument relies on the Stein-Chen method (see Chen (1975)) and Dynkin's formula, exploiting the particularly nice properties of the solutions to the Stein-Chen equation for one point subsets of $\mathbb{Z}_{+}$.

\subsection{Preliminaries}

For each $n \in \mathbb{N}$, let $Z_{n}(t), t \geq 0$, be an irreducible, continuous-time pure-jump Markov process taking values in $\mathbb{Z}$, with transition rates given by

$$
i \rightarrow i+j \quad \text { at rate } n \lambda_{j}\left(\frac{i}{n}\right), \quad i \in \mathbb{Z}, j \in \mathbb{Z} \backslash\{0\},
$$

where the $\lambda_{j}(\cdot)$ are prescribed functions on $\mathbb{R}$. We then define the 'overall jump rate' of the process $n^{-1} Z_{n}$ at $z \in n^{-1} \mathbb{Z}$ by

$$
\Lambda(z):=\sum_{j \in \mathbb{Z} \backslash\{0\}} \lambda_{j}(z)
$$

its 'average growth rate' by

$$
F(z):=\sum_{j \in \mathbb{Z} \backslash\{0\}} j \lambda_{j}(z),
$$

and its ‘quadratic variation' function by $n^{-1} \sigma^{2}(z)$, where

$$
\sigma^{2}(z)=\sum_{j \in \mathbb{Z} \backslash\{0\}} j^{2} \lambda_{j}(z),
$$

assumed to be finite for all $z \in \mathbb{R}$.

We make the following assumptions on the functions $\lambda_{j}$. The first requires a unique attractive equilibrium for the deterministic drift process.

(A1) There exists a unique $c$ satisfying $F(c)=0$; furthermore, $F^{\prime}(c)<0$ and, for any $\eta>0$, $\mu_{\eta}:=\inf _{|z-c| \geq \eta}|F(z)|>0$.

The next assumption controls the global behavior of the transition functions $\lambda_{j}$, limiting their growth away from the equilibrium $c$, as well as bounding a moment of the jump distribution. The condition on $\lambda_{1}$ is sufficient to exclude, for instance, processes confined to the even integers, for which our results would not hold.

(A2) (a) For each $j \in \mathbb{Z} \backslash\{0\}$, there exists $c_{j} \geq 0$ such that

$$
\lambda_{j}(z) \leq c_{j}(1+|z-c|), \quad z \in \mathbb{R}
$$

where the $c_{j}$ are such that, for some $0<\alpha \leq 1, \sum_{j \in \mathbb{Z} \backslash\{0\}}|j|^{2+\alpha} c_{j}=: s_{\alpha}<\infty$.

(b) For some $\lambda^{0}>0, \lambda_{1}(z) \geq 2 \lambda^{0}, z \in \mathbb{R}$.

We also require some smoothness and uniformity of the functions $\lambda_{j}$ near $c$. 
(A3) There exist $\varepsilon>0$ and $0<\delta \leq 1$ and a set $J \subset \mathbb{Z} \backslash\{0\}$ such that $\inf _{|z-c| \leq \delta} \lambda_{j}(z) \geq \varepsilon \lambda_{j}(c)>0, \quad j \in J ; \quad \lambda_{j}(z)=0 \quad$ for all $|z-c| \leq \delta, j \notin J$.

(A4) (a) For each $j \in J, \lambda_{j}$ is of class $C^{2}$ on $|z-c| \leq \delta$.

(b) For $\delta$ as in (A3),

$$
L_{1}:=\sup _{j \in J} \frac{\left\|\lambda_{j}^{\prime}\right\|_{\delta}}{\lambda_{j}(c)}<\infty, \quad L_{2}:=\sup _{j \in J} \frac{\left\|\lambda_{j}^{\prime \prime}\right\|_{\delta}}{|j| \lambda_{j}(c)}<\infty,
$$

where $\|f\|_{\delta}:=\sup _{|z-c| \leq \delta}|f(z)|$.

These assumptions imply in particular that the functions $\Lambda$ and $F$ are of class $C^{2}$ on $|z-c| \leq \delta$, that $\sigma^{2}$ is of class $C^{1}$ there, and that their derivatives can be obtained by differentiating inside their defining sums.

In Socoll and Barbour (2009), under assumptions (A1)-(A4), it was shown that the process $Z_{n}$ has an equilibrium distribution $\Pi_{n}$, and that $\hat{\Pi}_{n}:=\Pi_{n} * \delta_{-\lfloor n c\rfloor}$ satisfies

$$
d_{\mathrm{TV}}\left(\hat{\Pi}_{n}, \widehat{\mathrm{Po}}\left(n v_{c}\right)\right)=O\left(n^{-\alpha / 2}\right),
$$

where $v_{c}:=\sigma^{2}(c) /\left\{-2 F^{\prime}(c)\right\}$ and $\widehat{\operatorname{Po}}\left(n v_{c}\right)$ denotes the centered Poisson distribution

$$
\widehat{\operatorname{Po}}\left(n v_{c}\right):=\operatorname{Po}\left(n v_{c}\right) * \delta_{-\left\lfloor n v_{c}\right\rfloor} ;
$$

here, $\delta_{r}$ denotes the point mass at $r$, and ' $*$ ' denotes convolution. In this paper we prove the complementary local limit approximation.

Theorem 1.1. Under assumptions (A1)-(A4), there exists a constant $C>0$ such that

$$
\sup _{k \in \mathbb{Z}}\left|\hat{\Pi}_{n}(k)-\widehat{\operatorname{Po}}\left(n v_{c}\right)\{k\}\right| \leq C n^{-(\alpha+1) / 2} \sqrt{\log n} .
$$

This theorem shows that, even at the level of point probabilities, the approximation to $\Pi_{n} * \delta_{-\lfloor n c\rfloor}$ provided by the centered Poisson distribution $\widehat{\operatorname{Po}}\left(n v_{c}\right)$ is almost exactly the best that could be expected.

The proof is based on exploiting the equation

$$
\mathrm{E}\left\{\mathcal{A}_{n} h\right\}\left(Z_{n}\right)=0,
$$

where $\mathcal{A}_{n}$ denotes the infinitesimal generator of $Z_{n}$ :

$$
\left(\mathcal{A}_{n} h\right)(i):=\sum_{j \in \mathbb{Z} \backslash\{0\}} n \lambda_{j}\left(\frac{i}{n}\right)(h(i+j)-h(i)), \quad i \in \mathbb{Z},
$$

and where, here and subsequently, the quantity $Z_{n}$, when appearing without a time argument, is to be interpreted in such expressions as being a random variable having equilibrium distribution $\Pi_{n}$. Equation (1.1) is a manifestation of Dynkin's formula, and it holds under rather mild conditions on $h$; see Hamza and Klebaner (1995). The first step is to approximate $\mathcal{A}_{n} h$ by replacing $h(i+j)-h(i)$ with the first terms of its Newton expansion:

$$
h(i+j)-h(i) \approx j \Delta h(i)+\frac{1}{2} j^{2} \Delta^{2} h(i),
$$


where $\Delta f(i):=f(i+1)-f(i)$ and $\Delta^{2} f:=\Delta(\Delta f)$. Substituting (1.3) into (1.2) gives

$$
\left(\mathcal{A}_{n} h\right)(i) \approx n F\left(\frac{i}{n}\right) g_{h}(i)+\frac{1}{2} n \sigma^{2}\left(\frac{i}{n}\right) \Delta g_{h}(i),
$$

where we write $g_{h}$ for $\Delta h$; a precise version is given in Lemma 3.1, below. Then, considering arguments $i=\lfloor n c\rfloor+j$ with $j / n$ small-the values to be expected in equilibrium-and approximating $F(i / n)$ and $\sigma^{2}(i / n)$ near $c$ (where $F(c)=0$ ), we obtain

$$
\left(\mathcal{A}_{n} h\right)(i) \approx j F^{\prime}(c) g_{h}(j+\lfloor n c\rfloor)+\frac{1}{2} n \sigma^{2}(c) \Delta g_{h}(j+\lfloor n c\rfloor) .
$$

Dividing through by $\left|F^{\prime}(c)\right|$ yields the Stein operator for the centered Poisson distribution $\widehat{\mathrm{Po}}\left(n v_{c}\right)$, applied to the function $g_{h}(\cdot+\lfloor n c\rfloor)$. This indicates that $\widehat{\mathrm{Po}}\left(n v_{c}\right)$ is the appropriate approximation for the centered equilibrium distribution $\hat{\Pi}_{n}$. The rest of the paper gives the argument necessary to dispose of the errors involved in this chain of approximations.

\section{Differences of point probabilities}

As an essential step in proving Theorem 1.1, we first need to show that the differences between the successive point probabilities of $\Pi_{n}$ are suitably small. The bound that we achieve is of order $O\left(n^{-1} \sqrt{\log n}\right)$. In order to prove this result, we begin with two lemmas. The first states that, for any $U \geq 1$, the distribution of $Z_{n}(U)$ has point probabilities which are uniformly of order $O\left(n^{-1 / 2}\right)$, if $Z_{n}(0)$ is close enough to $n c$.

Lemma 2.1. Under assumptions (A1)-(A4), for any $U \geq 1$, there exists $C_{2.1}(U)<\infty$ such that

$$
\sup _{k \in \mathbb{Z}|i-n c| \leq n \delta / 2} \sup _{\mid-1} \mathrm{P}\left[Z_{n}(U)=k \mid Z_{n}(0)=i\right] \leq C_{2.1}(U) n^{-1 / 2} .
$$

Proof. Note that, for any integer-valued random variable $X$,

$$
\sup _{k \in \mathbb{Z}} \mathrm{P}[X=k]=\sup _{k \in \mathbb{Z}}\{\mathrm{P}[X \leq k]-\mathrm{P}[X+1 \leq k]\} \leq d_{\mathrm{TV}}\left\{\mathcal{L}(X), \mathcal{L}(X) * \delta_{1}\right\},
$$

where $\mathcal{L}(X)$ denotes the distribution of $X$. Taking $X=Z_{n}(U)$ and applying Lemma A.2, below, completes the proof.

The next lemma shows that the differences between successive point probabilities of $Z_{n}(U)$ are uniformly close, to order $O\left(n^{-1} \sqrt{\log n}\right)$, for a large range of values of $Z_{n}(0)$ and for a particular choice of $U \geq 1$. This is the result that we shall then be able to extend to the equilibrium distribution $\Pi_{n}$. For $\Lambda^{*}:=\sup _{|z-c| \leq \delta / 2} \Lambda(z)$, we set

$$
U:=\max \left\{1, \frac{1}{2 \Lambda^{*}}\right\} \quad \text { and } \quad \delta_{1}^{\prime}:=\frac{\delta \exp \left\{-U\left\|F^{\prime}\right\|_{\delta}\right\}}{4} .
$$

Lemma 2.2. Under assumptions (A1)-(A4), and for $U$ and $\delta_{1}^{\prime}$ defined above, there exists $C_{2.2}<\infty$ such that

$$
\begin{aligned}
& \sup _{k \in \mathbb{Z}} \sup _{|i-n c| \leq n \delta_{1}^{\prime} / 2}\left|\mathrm{P}\left[Z_{n}(U)=k \mid Z_{n}(0)=i-1\right]-\mathrm{P}\left[Z_{n}(U)=k+1 \mid Z_{n}(0)=i\right]\right| \\
& \quad \leq C_{2.2} n^{-1} \sqrt{\log n} .
\end{aligned}
$$


Proof. We compare the probability measures $\mathcal{L}\left(\left(Z_{n}(u), 0 \leq u \leq U\right) \mid Z_{n}(0)=i-1\right) * \delta_{1}$ and $\mathcal{L}\left(\left(Z_{n}(u), 0 \leq u \leq U\right) \mid Z_{n}(0)=i\right)$ by examining the likelihood ratio of the processes $Z_{n}^{(1)} \stackrel{\mathrm{D}}{=}\left\{Z_{n} \mid Z_{n}(0)=i-1\right\}$ and $Z_{n}^{(2)} \stackrel{\mathrm{D}}{=}\left\{Z_{n} \mid Z_{n}(0)=i\right\}$ along paths with the same set of jumps $\left(j_{l}, l \geq 1\right)$ occurring at the same times $\left(t_{l}, l \geq 1\right)$. (Here ' $=$ ' denotes equality in distribution.) The process $Z_{n}^{(1)}$ starts from the state $i-1$; we write $z_{l}:=n^{-1}\left(i-1+\sum_{s=1}^{l} j_{s}\right)$ for the value of $n^{-1} Z_{n}^{(1)}$ at time $t_{l}, l \geq 0$, and $z(u):=z_{l}$ if $t_{l} \leq u<t_{l+1}$. The process $Z_{n}^{(2)}$ starts from the state $i$, and, thus, has the same paths as $Z_{n}^{(1)}+1$. Then, using the notation $v[0, \infty)$ to denote the function $(v(t), t \geq 0)$, the likelihood ratio of the two processes along the first $m$ steps of the path is given by

$$
\begin{aligned}
S_{m} & :=S_{m}(z[0, \infty)) \\
& :=S_{m}\left(z_{0}, z_{1} \ldots, z_{m} ; t_{1}, \ldots, t_{m}\right) \\
& :=\prod_{l=0}^{m-1}\left(\frac{\lambda_{j_{l+1}}\left(z_{l}+n^{-1}\right)}{\lambda_{j_{l+1}}\left(z_{l}\right)} \exp \left\{-n\left(\Lambda\left(z_{l}+n^{-1}\right)-\Lambda\left(z_{l}\right)\right)\left(t_{l+1}-t_{l}\right)\right\}\right) \\
& =: \prod_{l=0}^{m-1} V_{l} .
\end{aligned}
$$

Note that, since $|(1+x)(1+y)-1| \leq 3|x|+|y|$ if $|y| \leq 2$, and since $\left|\mathrm{e}^{t}-1\right| \leq 2|t|$ if $t \leq 1$, it follows that

$$
\left|V_{l}-1\right| \leq 3\left|\frac{\lambda_{j_{l+1}}\left(z_{l}+n^{-1}\right)}{\lambda_{j_{l+1}}\left(z_{l}\right)}-1\right|+2 n\left|\Lambda\left(z_{l}+n^{-1}\right)-\Lambda\left(z_{l}\right)\right|\left(t_{l+1}-t_{l}\right),
$$

provided that $n\left\{\Lambda\left(z_{l}\right)-\Lambda\left(z_{l}+n^{-1}\right)\right\}\left(t_{l+1}-t_{l}\right) \leq 1$.

Now, if $|z-c| \leq \delta / 2$ and $n^{-1} \leq \delta / 2$, it follows from assumptions (A3) and (A4) that

$$
\left|\frac{\lambda_{j}\left(z+n^{-1}\right)}{\lambda_{j}(z)}-1\right| \leq \frac{\left\|\lambda_{j}^{\prime}\right\|_{\delta}}{n \varepsilon \lambda_{j}(c)} \leq \frac{L_{1}}{n \varepsilon}, \quad\left|\frac{\Lambda\left(z+n^{-1}\right)}{\Lambda(z)}-1\right| \leq \frac{\left\|\Lambda^{\prime}\right\|_{\delta}}{n \varepsilon \Lambda(c)} \leq \frac{L_{1}}{n \varepsilon} .
$$

Hence, for all $n \geq 2 / \delta$, writing $e_{l+1}:=n \Lambda\left(z_{l}\right)\left(t_{l+1}-t_{l}\right)$, we have

$$
\left|V_{l}-1\right| \leq \frac{L_{1}}{n \varepsilon}\left(3+2 e_{l+1}\right),
$$

as long as

$$
\left|z_{l}-c\right| \leq \frac{\delta}{2} \quad \text { and either } \quad \Lambda\left(z_{l}\right) \leq \Lambda\left(z_{l}+n^{-1}\right) \quad \text { or } \quad e_{l+1} \leq \frac{n \varepsilon}{L_{1}} .
$$

Now consider the random likelihood ratio process

$$
\left(S_{m}\left(n^{-1} Z_{0}, n^{-1} Z_{1}, \ldots, n^{-1} Z_{m} ; \tau_{1}, \ldots, \tau_{m}\right), m \geq 0\right),
$$

where $\left(\tau_{l}, l \geq 0\right)$ denote the jump times of the process $Z_{n}^{(1)}$, and $Z_{l}:=Z_{n}^{(1)}\left(\tau_{l}\right), l \geq 0$, the sequence of states that it visits; also define $E_{l}:=n \Lambda\left(n^{-1} Z_{l-1}\right)\left(\tau_{l}-\tau_{l-1}\right)$. Then $S:=$ $\left(S_{m}, m \geq 0\right)$ is a martingale with mean 1 with respect to the filtration $g_{m}:=\sigma\left(Z_{0}, Z_{1}, \ldots, Z_{m}\right.$; $\left.\tau_{1}, \ldots, \tau_{m}\right), m \geq 0$. We shall, for technical reasons, work rather with another martingale $\tilde{S}$, which typically agrees with $S$ for a long time, but which satisfies the inequality

$$
\left|\tilde{S}_{m+1}-\tilde{S}_{m}\right| \leq \frac{2 L_{1}}{n \varepsilon}\left(3+2 E_{m+1}\right) \quad \text { for all } m \geq 0
$$


This we achieve by defining $\sigma:=\min \left\{\sigma_{r}, 1 \leq r \leq 3\right\}$, where

$$
\begin{gathered}
\sigma_{1}:=\inf \left\{l \geq 0: n\left|\Lambda\left(n^{-1}\left[Z_{l-1}+1\right]\right)-\Lambda\left(n^{-1} Z_{l-1}\right)\right|\left(\tau_{l}-\tau_{l-1}\right)>1\right\}, \\
\sigma_{2}:=\inf \left\{l \geq 0: S_{l}>2\right\}, \quad \text { and } \quad \sigma_{3}:=\inf \left\{l \geq 0:\left|n^{-1} Z_{l}-c\right|>\frac{\delta}{2}\right\},
\end{gathered}
$$

and then setting

$$
\tilde{S}_{m}:=S_{m \wedge \sigma} C_{m, \sigma_{1}}
$$

where

$$
C_{m, l}:= \begin{cases}\frac{\mathrm{e}}{V_{l-1}} & \text { if } l \leq \min \left\{m, \sigma_{2}, \sigma_{3}\right\} \text { and } \Lambda\left(z_{l-1}\right)>\Lambda\left(z_{l-1}+n^{-1}\right) \\ 1 & \text { otherwise. }\end{cases}
$$

Note that the only effect of the factor $C_{m, \sigma_{1}}$ is to multiply $\tilde{S}$ by e instead of by $V_{\sigma_{1}-1}$ at time $\sigma_{1}$, if $\sigma_{1} \leq \min \left\{\sigma_{2}, \sigma_{3}\right\}$ and $\Lambda\left(z_{\sigma_{1}-1}\right)>\Lambda\left(z_{\sigma_{1}-1}+n^{-1}\right)$. The value $\mathrm{e}$ is chosen so that the martingale property is preserved; and the modification also ensures that (2.4) is still satisfied at time $\sigma_{1}$, since $2(\mathrm{e}-1)$ is no larger that $4 L_{1} E_{\sigma_{1}} /(n \varepsilon)$, because, at time $\sigma_{1}$,

$$
\begin{aligned}
1 & <n\left|\Lambda\left(z_{\sigma_{1}-1}+n^{-1}\right)-\Lambda\left(z_{\sigma_{1}-1}\right)\right|\left(\tau_{\sigma_{1}}-\tau_{\sigma_{1}-1}\right) \\
& =E_{\sigma_{1}}\left|\frac{\Lambda\left(z_{\sigma_{1}-1}+n^{-1}\right)}{\Lambda\left(z_{\sigma_{1}-1}\right)}-1\right| \\
& \leq E_{\sigma_{1}} \frac{L_{1}}{n \varepsilon}
\end{aligned}
$$

in view of (2.3).

Now, from (2.4), and since also, by the strong Markov property, the conditional distribution $\mathcal{L}\left(E_{l+1} \mid g_{l}\right)$ is the standard exponential $\exp (1)$ distribution for each $l$, the process $\tilde{S}$ satisfies the conditions of the variant of the bounded differences inequality for martingales given in Barbour (2008, Lemma 4.1), from which it follows that

$$
\mathrm{P}\left[\left|\tilde{S}_{m}-1\right|>C \frac{L_{1} \sqrt{m \log m}}{n \varepsilon} \mid Z_{n}(0)=i-1\right] \leq 2 \exp \left\{-\frac{3 C \log m}{928}\right\}
$$

for any $m$ such that $\sqrt{m / \log m} \geq 135 C / 236$. In particular, recalling (2.2), for $m=m(n):=$ $\left\lceil 2 n \Lambda^{*} U\right\rceil$, we have

$$
\mathrm{P}\left[\left|\tilde{S}_{m(n)}-1\right|>C \frac{L_{1} \sqrt{m(n) \log m(n)}}{n \varepsilon} \mid Z_{n}(0)=i-1\right] \leq 2 n^{-3},
$$

if we take $C:=928$, as long as $n \geq \mathrm{e}$ and

$$
\frac{n}{\log n} \geq 540^{2}
$$

Introducing the notation $\mathrm{P}_{s}$ to denote $\mathrm{P}\left[\cdot \mid Z_{n}(0)=s\right]$; defining $M_{n}(U):=\min \left\{l: \tau_{l}>U\right\}$, we would now like to use the equation

$$
\mathrm{P}_{i}\left[Z_{n}(U)=k+1\right]-\mathrm{P}_{i-1}\left[Z_{n}(U)+1=k+1\right]=\mathrm{E}_{i-1}\left(\left(S_{M_{n}(U)}-1\right) \mathbf{1}\left[Z_{n}(U)=k\right]\right),
$$


together with the control over $\left|\tilde{S}_{m(n)}-1\right|$ from (2.6), to establish the inequality that we are looking for. However, the fact that $S_{M_{n}(U)}$ may be unbounded makes this complicated. Instead, we argue from

$$
\begin{gathered}
\mathrm{E}_{i}\left\{\mathbf{1}\left[Z_{n}(U)=k+1\right] g\left(Z_{n}[0, \infty)\right)\right\}-\mathrm{E}_{i-1}\left\{\mathbf{1}\left[Z_{n}(U)+1=k+1\right] g\left(\left(Z_{n}+1\right)[0, \infty)\right)\right\} \\
=\mathrm{E}_{i-1}\left\{\left(S_{M_{n}(U)}-1\right) \mathbf{1}\left[Z_{n}(U)+1=k+1\right] g\left(\left(Z_{n}+1\right)[0, \infty)\right)\right\},
\end{gathered}
$$

where

$$
g((Z+1)[0, \infty)):=\mathbf{1}\left[S_{m_{n}\left(U, t_{\mathbb{N}_{0}}\right)}\left(n^{-1} Z[0, \infty)\right) \leq \bar{s}\right] \mathbf{1}\left[m_{n}\left(U, t_{\mathbb{N}_{0}}\right) \leq m(n)\right]
$$

for suitably chosen $\bar{s}$, with $m_{n}\left(U, t_{\mathbb{N}_{0}}\right):=\min \left\{l: t_{l}>U\right\}$. More explicitly, we have

$$
g\left(\left(Z_{n}+1\right)[0, \infty)\right)=\mathbf{1}\left[S_{M_{n}(U)} \leq \bar{s}\right] \mathbf{1}\left[M_{n}(U) \leq m(n)\right] .
$$

We start by showing that the value of $g\left(\left(Z_{n}+1\right)[0, \infty)\right)$ is 1 with high probability, so that $(2.8)$ closely approximates the difference that we wish to bound.

First, if

$$
\max _{1 \leq l \leq m_{n}\left(U, t_{\mathbb{N}_{0}}\right)}\left|z_{l}-c\right| \leq \frac{\delta}{2}, \quad m_{n}\left(U, t_{\mathbb{N}_{0}}\right) \leq m(n) \quad \text { and } \quad t_{m_{n}\left(U, t_{\mathbb{N}_{0}}\right)}-U \leq 1,
$$

it follows from (2.3) and the definition of $m(n)$ that

$$
S_{m} \leq\left(1+\frac{L_{1}}{n \varepsilon}\right)^{m} \exp \left\{\left\|\Lambda^{\prime}\right\|_{\delta} t_{m}\right\} \leq \exp \left\{1+2 \Lambda^{*} U\left(\frac{L_{1}}{\varepsilon}\right)+\left\|\Lambda^{\prime}\right\|_{\delta}(U+1)\right\}=: \bar{s}
$$

for all $m \leq m_{n}\left(U, t_{\mathbb{N}_{0}}\right)$. Hence, with this definition of $\bar{s}, g\left(\left(Z_{n}+1\right)[0, \infty)\right)=1$ on $A_{3} \cap A_{4} \cap A_{5}$, where $A_{3}:=\left\{\sigma_{3} \geq M_{n}(U)\right\}, A_{4}:=\left\{M_{n}(u) \leq m(n)\right\}$, and $A_{5}:=\left\{t_{M_{n}(U)}-U \leq 1\right\}$. Now, from Lemma A.1, for all $|i-n c| \leq n \delta_{1}^{\prime}$, as defined in (2.2), we have

$$
\mathrm{P}_{i-1}\left[A_{3}^{\mathrm{c}}\right]=\mathrm{P}_{i-1}\left[\sup _{0 \leq u \leq U}\left|n^{-1} Z_{n}(u)-c\right|>\frac{\delta}{2}\right] \leq n^{-1} K_{U, \delta / 2} .
$$

Then, by the Chernoff inequality (see Chung and Lu (2006, Theorem 4)),

$$
\begin{aligned}
\mathrm{P}_{i-1}\left[A_{4}^{\mathrm{c}} \cap A_{3}\right] & =\mathrm{P}_{i-1}\left[\left\{\tau_{m(n)} \leq U\right\} \cap A_{3}\right] \\
& \leq \operatorname{Po}\left(n \Lambda^{*} U\right)\left\{\left(2 n \Lambda^{*} U, \infty\right)\right\} \\
& \leq \exp \left\{-\frac{n \Lambda^{*} U}{3}\right\},
\end{aligned}
$$

and $\mathrm{P}_{i-1}\left[A_{5}^{\mathrm{c}}\right] \leq \exp \left\{-2 n \lambda^{0}\right\}$ is immediate from assumption (A2). Hence, we have proved that

$$
\mathrm{P}_{i-1}\left[g\left(\left(Z_{n}+1\right)[0, \infty)\right)=0\right] \leq \eta_{1}(n),
$$

where $\eta_{1}(n) \leq C n^{-1}$ for some $C<\infty$. The same bound also holds for the probability $\mathrm{P}_{i}\left[g\left(Z_{n}[0, \infty)\right)=0\right]$, if we restrict $i$ further to the set $|i-n c| \leq n \delta_{1}^{\prime} / 2$, provided that $\delta_{1}^{\prime} / 2 \geq n^{-1}$, because $S(z)$ is now computed with arguments shifted by $n^{-1}$. Thus, and from (2.8) and (2.9), we conclude that

$$
\begin{aligned}
& \left|\mathrm{P}_{i}\left[Z_{n}(U)=k+1\right]-\mathrm{P}_{i-1}\left[Z_{n}(U)=k\right]\right| \\
& \quad \leq\left|\mathrm{E}_{i-1}\left\{\left(S_{M_{n}(U)}-1\right) \mathbf{1}\left[Z_{n}(U)=k\right] \mathbf{1}\left[S_{M_{n}(U)} \leq \bar{s}\right] \mathbf{1}\left[A_{4}\right]\right\}\right|+2 \eta_{1}(n) .
\end{aligned}
$$


However, it is immediate that

$$
\begin{aligned}
\mid \mathrm{E}_{i-1}\{ & \left.\left(S_{M_{n}(U)}-1\right) \mathbf{1}\left[Z_{n}(U)=k\right] \mathbf{1}\left[S_{M_{n}(U)} \leq \bar{s}\right] \mathbf{1}\left[A_{4}\right]\right\} \mid \\
\leq & \left|\mathrm{E}_{i-1}\left\{\left(\tilde{S}_{M_{n}(U)}-1\right) \mathbf{1}\left[Z_{n}(U)=k\right] \mathbf{1}\left[S_{M_{n}(U)} \leq \bar{s}\right] \mathbf{1}\left[A_{4}\right] \mathbf{1}\left[\tilde{S}_{M_{n}(U)}=S_{M_{n}(U)}\right]\right\}\right| \\
\quad & +\bar{s} \mathrm{P}_{i-1}\left[\left\{\tilde{S}_{M_{n}(U)} \neq S_{M_{n}(U)}\right\} \cap A_{4}\right],
\end{aligned}
$$

and, since $Z_{n}(U)$ is $g_{M_{n}(U)}$-measurable, it follows from the martingale property that

$$
\begin{aligned}
& \left|\mathrm{E}_{i-1}\left\{\left(\tilde{S}_{M_{n}(U)}-1\right) \mathbf{1}\left[Z_{n}(U)=k\right] \mathbf{1}\left[S_{M_{n}(U)} \leq \bar{s}\right] \mathbf{1}\left[A_{4}\right] \mathbf{1}\left[\tilde{S}_{M_{n}(U)}=S_{M_{n}(U)}\right]\right\}\right| \\
& \quad=\left|\mathrm{E}_{i-1}\left\{\left(\tilde{S}_{m(n)}-1\right) \mathbf{1}\left[Z_{n}(U)=k\right] \mathbf{1}\left[S_{M_{n}(U)} \leq \bar{s}\right] \mathbf{1}\left[A_{4}\right] \mathbf{1}\left[\tilde{S}_{M_{n}(U)}=S_{M_{n}(U)}\right]\right\}\right| \\
& \quad \leq \frac{C L_{1}}{n \varepsilon} \sqrt{m(n) \log m(n)} \mathrm{P}_{i-1}\left[Z_{n}(U)=k\right]+(2 \mathrm{e}-1) n^{-3},
\end{aligned}
$$

the last inequality holding for all $n \geq$ e satisfying (2.7), by (2.6).

It remains now first to bound

$$
\mathrm{P}_{i-1}\left[\left\{\tilde{S}_{M_{n}(U)} \neq S_{M_{n}(U)}\right\} \cap A_{4}\right] \leq \mathrm{P}_{i-1}\left[A_{4} \cap\left\{\bigcup_{l=1}^{3} A_{l}^{\mathrm{c}}\right\}\right],
$$

where $A_{1}:=\left\{\sigma_{1}>M_{n}(U)\right\}$ and $A_{2}:=\left\{\sigma_{2}>M_{n}(U)\right\}$. We already have a bound for $\mathrm{P}_{i-1}\left[A_{3}^{\mathrm{c}}\right],(2.10)$. Then, from (2.5) and the definition of $E_{l}$, and using (2.3), we have

$$
A_{1}^{\mathrm{c}} \cap A_{3} \cap A_{4} \subset \bigcup_{l=1}^{m(n)}\left\{\frac{L_{1} E_{l}}{n \varepsilon}>1\right\},
$$

so that

$$
\mathrm{P}_{i-1}\left[A_{1}^{\mathrm{c}} \cap A_{3} \cap A_{4}\right] \leq m(n) \exp \left\{-\frac{n \varepsilon}{L_{1}}\right\} .
$$

Finally, on the set $A_{2}^{\mathrm{c}} \cap A_{1} \cap A_{3} \cap A_{4}$, we then note that

$$
\left|\tilde{S}_{m(n)}-1\right|>C \frac{L_{1} \sqrt{m(n) \log m(n)}}{n \varepsilon}
$$

for all $n \geq \max \left\{3,2 \Lambda^{*}\right\}$ such that $n / \log n>3\left(L_{1} / \varepsilon\right)^{2}$, implying that, for $n$ that also satisfy (2.7),

$$
\mathrm{P}_{i-1}\left[A_{2}^{\mathrm{c}} \cap A_{1} \cap A_{3} \cap A_{4}\right] \leq 2 n^{-3},
$$

from (2.6). Combining these bounds, and also noting that, from Lemma 2.1,

$$
\mathrm{P}_{i-1}\left[Z_{n}(U)=k\right] \leq \frac{C_{2.1}(U)}{\sqrt{n}}
$$

for all $|i-n c| \leq n \delta_{1}^{\prime}$, the lemma is proved.

Theorem 2.1. Under assumptions (A1)-(A4), there exists a constant $C_{2.1}^{\star}>0$ such that

$$
\sup _{k \in \mathbb{Z}}\left|\Pi_{n}(k)-\Pi_{n}(k+1)\right| \leq C_{2.1}^{\star} n^{-1} \sqrt{\log n}
$$


Proof. Fix $U$ as in (2.2). Since $\Pi_{n}$ is the equilibrium distribution of $Z_{n}$, it is in particular true that

$$
\begin{aligned}
\left|\Pi_{n}(k)-\Pi_{n}(k+1)\right|= & \left|\sum_{i \in \mathbb{Z}} \Pi_{n}(i) \mathrm{P}_{i}\left[Z_{n}(U)=k\right]-\sum_{i \in \mathbb{Z}} \Pi_{n}(i) \mathrm{P}_{i}\left[Z_{n}(U)=k+1\right]\right| \\
\leq & \sum_{i \in \mathbb{Z}} \Pi_{n}(i-1)\left|\mathrm{P}_{i-1}\left[Z_{n}(U)=k\right]-\mathrm{P}_{i}\left[Z_{n}(U)=k+1\right]\right| \\
& +\sum_{i \in \mathbb{Z}}\left|\Pi_{n}(i-1)-\Pi_{n}(i)\right| \mathrm{P}_{i}\left[Z_{n}(U)=k+1\right] .
\end{aligned}
$$

With $\delta_{1}^{\prime}$ as in (2.2), note that we can write

$$
\begin{aligned}
\sum_{i \in \mathbb{Z}} & \Pi_{n}(i-1)\left|\mathrm{P}_{i-1}\left[Z_{n}(U)=k\right]-\mathrm{P}_{i}\left[Z_{n}(U)=k+1\right]\right| \\
& \leq \Pi_{n}\left(\left|Z_{n}+1-n c\right|>n \delta_{1}^{\prime}\right)+\sup _{|i-n c| \leq n \delta_{1}^{\prime}}\left|\mathrm{P}_{i-1}\left[Z_{n}(U)=k\right]-\mathrm{P}_{i}\left[Z_{n}(U)=k+1\right]\right|,
\end{aligned}
$$

and that

$$
\begin{aligned}
& \sum_{i \in \mathbb{Z}}\left|\Pi_{n}(i-1)-\Pi_{n}(i)\right| \mathrm{P}_{i}\left[Z_{n}(U)=k+1\right] \\
& \leq \Pi_{n}\left(\left|Z_{n}+1-n c\right|>n \delta_{1}^{\prime}\right)+\Pi_{n}\left(\left|Z_{n}-n c\right|>n \delta_{1}^{\prime}\right) \\
&+\sup _{|i-n c| \leq n \delta_{1}^{\prime}} \mathrm{P}_{i}\left[Z_{n}(U)=k+1\right] 2 d_{\mathrm{TV}}\left\{\Pi_{n}, \Pi_{n} * \delta_{1}\right\}
\end{aligned}
$$

By applying the result of Corollary A.1 three times we obtain

$$
\begin{aligned}
\sup _{k \in \mathbb{Z}}\left|\Pi_{n}(k)-\Pi_{n}(k+1)\right| & \\
\leq & O\left(n^{-1}\right)+\sup _{k \in \mathbb{Z}} \sup _{|i-n c| \leq n \delta_{1}^{\prime}}\left|\mathrm{P}_{i-1}\left[Z_{n}(U)=k\right]-\mathrm{P}_{i}\left[Z_{n}(U)=k+1\right]\right| \\
& +\sup _{k \in \mathbb{Z}|i-n c| \leq n \delta_{1}^{\prime}} \sup _{i}\left[Z_{n}(U)=k+1\right] 2 d_{\mathrm{TV}}\left\{\Pi_{n}, \Pi_{n} * \delta_{1}\right\} \\
= & : O\left(n^{-1}\right)+\eta_{1 n}+\eta_{2 n} .
\end{aligned}
$$

The quantity $\eta_{1 n}$ is of order $O\left(n^{-1} \sqrt{\log n}\right)$, in view of Lemma 2.2; and Lemma 2.1 and Theorem A.2 together give the bound

$$
\eta_{2 n} \leq C_{2.1}(U) n^{-1 / 2} C_{A .2} n^{-1 / 2}=O\left(n^{-1}\right) .
$$

This completes the proof of the theorem.

\section{Local limit approximation for the equilibrium distribution}

As outlined at the end of the preliminaries, we start from the fact that $\mathrm{E}\left\{\mathcal{A}_{n} h\right\}\left(Z_{n}\right)=0$ for many functions $h$, and transform it using Stein's method into a statement concerning the closeness of $\Pi_{n}$ to a suitably translated Poisson distribution. The first step is to recall a result from Socoll and Barbour (2009), which shows that $\mathcal{A}_{n}$ can be expressed in a form which is closer to that of the desired Stein operator. 
Lemma 3.1. (Socoll and Barbour (2009, Lemma 1.1).) Suppose that $\sigma^{2}(z)<\infty$ for all $z \in \mathbb{R}$. Then, for any function $h: \mathbb{Z} \rightarrow \mathbb{R}$ with bounded differences, we have

$$
\left(\mathcal{A}_{n} h\right)(i)=\frac{n}{2} \sigma^{2}\left(\frac{i}{n}\right) \nabla g_{h}(i)+n F\left(\frac{i}{n}\right) g_{h}(i)+E_{n}\left(g_{h}, i\right),
$$

where $\nabla f(i):=f(i)-f(i-1)$ and $g_{h}(i):=\nabla h(i+1)$, and, for any $i \in \mathbb{Z}$,

$$
E_{n}(g, i):=-\frac{n}{2} F\left(\frac{i}{n}\right) \nabla g(i)+\sum_{j \geq 2} a_{j}(g, i) n \lambda_{j}\left(\frac{i}{n}\right)-\sum_{j \geq 2} b_{j}(g, i) n \lambda_{-j}\left(\frac{i}{n}\right),
$$

with

$$
\begin{aligned}
& a_{j}(g, i):=-\left(\begin{array}{l}
j \\
2
\end{array}\right) \nabla g(i)+\sum_{k=1}^{j-1} k \nabla g(i+j-k)=\sum_{k=2}^{j}\left(\begin{array}{l}
k \\
2
\end{array}\right) \nabla^{2} g(i+j-k+1), \\
& b_{j}(g, i):=\left(\begin{array}{l}
j \\
2
\end{array}\right) \nabla g(i)-\sum_{k=1}^{j-1} k \nabla g(i-j+k)=\sum_{k=2}^{j}\left(\begin{array}{l}
k \\
2
\end{array}\right) \nabla^{2} g(i-j+k) .
\end{aligned}
$$

Since $F(c)=0$, we note that, for small $i / n,\left\{-F^{\prime}(c)\right\}^{-1}\left(\mathcal{A}_{n} h\right)(i+\lfloor n c\rfloor)$ is close to

$$
\frac{1}{-F^{\prime}(c)} \frac{n}{2} \sigma^{2}(c) \Delta g_{h}^{*}(i)-\left(i-\left\langle n v_{c}\right\rangle\right) g_{h}^{*}(i)=n v_{c} \Delta g_{h}^{*}(i)-\left(i-\left\langle n v_{c}\right\rangle\right) g_{h}^{*}(i)
$$

for $g_{h}^{*}(i):=g_{h}(i+\lfloor n c\rfloor)$, where $\left\langle n v_{c}\right\rangle=n v_{c}-\left\lfloor n v_{c}\right\rfloor$ denotes the fractional part of $n v_{c}$. This is the Stein operator for the centered Poisson distribution $\widehat{\operatorname{Po}}\left(n v_{c}\right)$ (see Röllin (2005)), acting on the function $g_{h}^{*}$. Combining this observation with (1.1) and writing $Y_{n}=Z_{n}-\lfloor n c\rfloor$ yields

$$
\begin{aligned}
0 & =\left\{-F^{\prime}(c)\right\}^{-1} \mathrm{E}\left\{\left(\mathcal{A}_{n} h\right)\left(Y_{n}+\lfloor n c\rfloor\right)\right\} \\
& =\mathrm{E}\left\{n v_{c} \Delta g_{h}^{*}\left(Y_{n}\right)-\left(i-\left\langle n v_{c}\right\rangle\right) g_{h}^{*}\left(Y_{n}\right)\right\}+\mathrm{E}\left\{H\left(g_{h}^{*}, Y_{n}\right)\right\},
\end{aligned}
$$

say. If the error term $\mathrm{E}\left\{H\left(g_{h}^{*}, Y_{n}\right)\right\}$ can be controlled, then Stein's method leads easily to the approximation of $\mathcal{L}\left(Y_{n}\right)=\Pi_{n} * \delta_{-\lfloor n c\rfloor}$ by $\widehat{\operatorname{Po}}\left(n v_{c}\right)$. For the approximation of point probabilities, (3.1) needs to be analyzed for functions $g_{h}^{*}$ that are translates of the solutions to the Stein-Chen equation corresponding to single-point sets.

Carrying out this recipe, and examining the form of $H\left(g_{h}^{*}, Y_{n}\right)$, yields

$$
\begin{aligned}
& \sup _{r \in \mathbb{Z}}\left|\left(\Pi_{n}-\lfloor n c\rfloor\right)(r)-\widehat{\operatorname{Po}}\left(n v_{c}\right)(r)\right| \\
& \leq \frac{1}{-F^{\prime}(c)} \sup _{r \in \mathbb{Z}}\left|\mathrm{E}\left\{R\left(n, r ; Y_{n}\right)\right\}\right|+\sup _{r \in \mathbb{Z}} n v_{c}\left|\mathrm{E}\left\{\nabla^{2} \tilde{g}_{n v_{c}, r}\left(Y_{n}+1\right)\right\}\right| \\
&+\sup _{r \in \mathbb{P}}\left(n v_{c}\right)\{r\} \mathrm{P}\left[Y_{n}<-\left\lfloor n v_{c}\right\rfloor\right] \\
&:= R_{n 1}+R_{n 2}+R_{n 3},
\end{aligned}
$$

say, where

$$
\begin{aligned}
R\left(n, r ; Y_{n}\right):= & \frac{n}{2}\left[\sigma^{2}\left(\frac{Y_{n}+\lfloor n c\rfloor}{n}\right)-\sigma^{2}(c)\right] \nabla \tilde{g}_{n v_{c}, r}\left(Y_{n}\right) \\
& +n\left[F\left(\frac{Y_{n}+\lfloor n c\rfloor}{n}\right)-F(c)-\frac{Y_{n}}{n} F^{\prime}(c)\right] \tilde{g}_{n v_{c}, r}\left(Y_{n}\right) \\
& +F^{\prime}(c)\left\langle n v_{c}\right\rangle \tilde{g}_{n v_{c}, r}\left(Y_{n}\right)+E_{n}\left(\tilde{g}_{n v_{c}, r}, Y_{n}+\lfloor n c\rfloor\right),
\end{aligned}
$$


and the function $\tilde{g}_{n v_{c}, r}$ is given by

$$
\tilde{g}_{n v_{c}, r}(l):= \begin{cases}0 & \text { if } l<-\left\lfloor n v_{c}\right\rfloor \\ g_{n v_{c},\left\{r+\left\lfloor n v_{c}\right\rfloor\right\}}\left(l+\left\lfloor n v_{c}\right\rfloor\right) & \text { if } l \geq-\left\lfloor n v_{c}\right\rfloor .\end{cases}
$$

Here, for $A \subset \mathbb{Z}_{+}, g_{\mu, A}$ denotes the solution to the Stein-Chen equation

$$
\mathbf{1}_{A}(i)-\operatorname{Po}(\mu)\{A\}=\mu g_{\mu, A}(i+1)-i g_{\mu, A}(i), \quad i \geq 0 .
$$

We further split the last term of (3.3) into

$$
E_{n}\left(\tilde{g}_{n v_{c}, r}, Y_{n}+\lfloor n c\rfloor\right)=\sum_{l=1}^{7} E_{n l}\left(\tilde{g}_{n v_{c}, r}, Y_{n}+\lfloor n c\rfloor\right),
$$

with

$$
\begin{aligned}
& E_{n 1}\left(\tilde{g}_{n v_{c}, r}, Y_{n}+\lfloor n c\rfloor\right):=-\frac{n}{2}\left(F\left(\frac{Y_{n}+\lfloor n c\rfloor}{n}\right)-F(c)\right) \nabla \tilde{g}_{n v_{c}, r}\left(Y_{n}\right), \\
& E_{n 2}\left(\tilde{g}_{n v_{c}, r}, Y_{n}+\lfloor n c\rfloor\right):=\sum_{j=2}^{\lfloor\sqrt{n}\rfloor}\left(\sum_{k=2}^{j}\left(\begin{array}{l}
k \\
2
\end{array}\right) \nabla^{2} \tilde{g}_{n v_{c}, r}\left(Y_{n}+j-k+1\right)\right) n \lambda_{j}(c), \\
& E_{n 3}\left(\tilde{g}_{n v_{c}, r}, Y_{n}+\lfloor n c\rfloor\right):=\sum_{j=2}^{\lfloor\sqrt{n}\rfloor}\left(-\left(\begin{array}{l}
j \\
2
\end{array}\right) \nabla \tilde{g}_{n v_{c}, r}\left(Y_{n}\right)+\sum_{k=1}^{j-1} k \nabla \tilde{g}_{n v_{c}, r}\left(Y_{n}+j-k\right)\right) \\
& \times n\left\{\lambda_{j}\left(\frac{Y_{n}+\lfloor n c\rfloor}{n}\right)-\lambda_{j}(c)\right\}, \\
& E_{n 4}\left(\tilde{g}_{n v_{c}, r}, Y_{n}+\lfloor n c\rfloor\right):=\sum_{j \geq\lceil\sqrt{n}\rceil}\left(-\left(\begin{array}{l}
j \\
2
\end{array}\right) \nabla \tilde{g}_{n v_{c}, r}\left(Y_{n}\right)+\sum_{k=1}^{j-1} k \nabla \tilde{g}_{n v_{c}, r}\left(Y_{n}+j-k\right)\right) \\
& \times n \lambda_{j}\left(\frac{Y_{n}+\lfloor n c\rfloor}{n}\right), \\
& E_{n 5}\left(\tilde{g}_{n v_{c}, r}, Y_{n}+\lfloor n c\rfloor\right):=-\sum_{j=2}^{\lfloor\sqrt{n}\rfloor}\left(\sum_{k=2}^{j}\left(\begin{array}{l}
k \\
2
\end{array}\right) \nabla^{2} \tilde{g}_{n v_{c}, r}\left(Y_{n}-j+k\right)\right) n \lambda_{-j}(c), \\
& E_{n 6}\left(\tilde{g}_{n v_{c}, r}, Y_{n}+\lfloor n c\rfloor\right):=-\sum_{j=2}^{\lfloor\sqrt{n}\rfloor}\left(\left(\begin{array}{l}
j \\
2
\end{array}\right) \nabla \tilde{g}_{n v_{c}, r}\left(Y_{n}\right)-\sum_{k=1}^{j-1} k \nabla \tilde{g}_{n v_{c}, r}\left(Y_{n}-j+k\right)\right) \\
& \times n\left\{\lambda_{-j}\left(\frac{Y_{n}+\lfloor n c\rfloor}{n}\right)-\lambda_{-j}(c)\right\}, \\
& E_{n 7}\left(\tilde{g}_{n v_{c}, r}, Y_{n}+\lfloor n c\rfloor\right):=-\sum_{j \geq\lceil\sqrt{n}\rceil}\left(\left(\begin{array}{l}
j \\
2
\end{array}\right) \nabla \tilde{g}_{n v_{c}, r}\left(Y_{n}\right)-\sum_{k=1}^{j-1} k \nabla \tilde{g}_{n v_{c}, r}\left(Y_{n}-j+k\right)\right) \\
& \times n \lambda_{-j}\left(\frac{Y_{n}+\lfloor n c\rfloor}{n}\right) .
\end{aligned}
$$


Our strategy for proving Theorem 1.1 is now to show that each of the terms $R_{n 1}, R_{n 2}$, and $R_{n 3}$ in (3.2) is of the desired order, $O\left(n^{-(\alpha+1) / 2} \sqrt{\log n}\right)$; clearly, the treatment of $R_{n 1}$, which involves all the detail of $E_{n}\left(\tilde{g}_{n v_{c}, r}, Y_{n}+\lfloor n c\rfloor\right)$, is to be the most laborious.

We begin by collecting some of the properties of the functions $\tilde{g}_{n v_{c}, r}$, defined in (3.4), that appear frequently. We write $\|f\|_{\infty}:=\sup _{i \in \mathbb{Z}}|f(i)|$ and $\|f\|_{l_{1}}:=\sum_{i \in \mathbb{Z}}|f(i)|$.

Lemma 3.2. We have the following estimates:

1. $\left\|\tilde{g}_{n v_{c}, r}\right\|_{\infty} \leq\left\|\Delta \tilde{g}_{n v_{c}, r}\right\|_{\infty} \leq 1 /\left(n v_{c}\right)$;

2. $\left\|\Delta \tilde{g}_{n v_{c}, r}(i)\right\|_{l_{1}} \leq 2 /\left(n v_{c}\right)$;

3. $\left\|\Delta^{2} \tilde{g}_{n v_{c}, r}(i)\right\|_{l_{1}} \leq 4 /\left(n v_{c}\right)$;

5. $\left|\left(i-\left\langle n v_{c}\right\rangle\right) \tilde{g}_{n v_{c}, r}(i)\right| \leq h(i)+\operatorname{Po}\left(n v_{c}\right)\left\{r+\left\lfloor n v_{c}\right\rfloor\right\}$;

6. $\left|\left(i-\left\langle n v_{c}\right\rangle\right) \Delta \tilde{g}_{n v_{c}, r}(i)\right| \leq h(i+1)+h(i)+1 /\left(n v_{c}\right)$,

where, in parts 4 and 5 we have $h(i) \geq 0$ for all $i$ and $\|h(i)\|_{l_{1}} \leq 3$.

Proof. For $i \leq-\left\lfloor n v_{c}\right\rfloor, \tilde{g}_{n v_{c}, r}(i)=0$; for $i>-\left\lfloor n v_{c}\right\rfloor$, we have $\tilde{g}_{n v_{c}, r}(i)=g_{\mu, s}(j)$, where $j=i+\left\lfloor n v_{c}\right\rfloor, \mu=n v_{c}$, and $s=r+\left\lfloor n v_{c}\right\rfloor$, and $g=g_{\mu, s}$ satisfies the Stein-Chen equation

$$
\mu g(j+1)-j g(j)=\mathbf{1}_{\{s\}}(j)-\operatorname{Po}(\mu)\{s\}, \quad j \geq 0 .
$$

Parts 1 and 2 now follow from the proof of Lemma 1.1.1 of Barbour et al. (1992), in which it was shown that the function $g_{\mu, s}$ is negative and strictly decreasing in $\{1,2, \ldots, s\}$, and positive and strictly decreasing in $\{s+1, s+2, \ldots\}$, with $\Delta g_{\mu, s}(s) \leq 1 /\left(n v_{c}\right)$. Part 3 is then immediate from part 2.

For part 4, using the notation above and (3.13), we have

$$
\begin{aligned}
\left(i-\left\langle n v_{c}\right\rangle\right) \tilde{g}_{n v_{c}, r}(i) & =(j-\mu) g_{\mu, s}(j) \\
& =\mu\left(g_{\mu, s}(j+1)-g_{\mu, s}(j)\right)-\mathbf{1}_{\{s\}}(j)+\operatorname{Po}(\mu)\{s\} .
\end{aligned}
$$

This implies that

$$
\left|\left(i-\left\langle n v_{c}\right\rangle\right) \tilde{g}_{n v_{c}, r}(i)\right| \leq\left\{\mu|\Delta g(j)|+\mathbf{1}_{\{s\}}(j)\right\}+\operatorname{Po}(\mu)\{s\},
$$

which, with part 2, proves part 4. It also follows immediately from (3.14) that

$$
\left|\left(i-\left\langle n v_{c}\right\rangle\right) \Delta \tilde{g}_{n v_{c}, r}(i)\right| \leq h(i+1)+h(i)+\left|\tilde{g}_{n v_{c}, r}(i+1)\right|,
$$

for the same function $h(i):=\left\{\mu|\Delta g(j)|+\mathbf{1}_{\{s\}}(j)\right\}$, and part 5 follows on applying part 1 .

As a result of these bounds, combined with Theorems 2.1 and A.2, we can establish two useful bounds on expectations of differences of the $\tilde{g}_{n v_{c}, r}\left(Y_{n}+\cdot\right)$, under the equilibrium distribution.

Lemma 3.3. For any $r, l \in \mathbb{Z}$, we have

1. $\mathrm{E}\left|\nabla \tilde{g}_{n v_{c}, r}\left(Y_{n}+l\right)\right| \leq \frac{2 C_{A .2}}{n^{3 / 2} v_{c}}$;

2. $\left|\mathrm{E}\left\{\nabla^{2} \tilde{g}_{n v_{c}, r}\left(Y_{n}+l\right)\right\}\right| \leq \frac{2 C_{2.1}^{\star}}{n^{2} v_{c}} \sqrt{\log n}$. 
Proof. For the first part, it is immediate that

$$
\mathrm{E}\left|\nabla \tilde{g}_{n v_{c}, r}\left(Y_{n}+l\right)\right| \leq \sup _{i^{\prime} \in \mathbb{Z}} \Pi_{n}\left(i^{\prime}\right) \sum_{i \in \mathbb{Z}}\left|\nabla \tilde{g}_{n v_{c}, r}(i)\right| .
$$

By part 2 of Lemma 3.2 and (2.1), this is bounded in turn by $d_{\mathrm{TV}}\left\{\Pi_{n}, \Pi_{n} * \delta_{1}\right\} 2 /\left(n v_{c}\right)$, and part 1 follows from Theorem A.2. For the second part,

$$
\begin{aligned}
\left|\mathrm{E}\left\{\nabla^{2} \tilde{g}_{n v_{c}, r}\left(Y_{n}+l\right)\right\}\right| & =\left|\sum_{i \in \mathbb{Z}} \nabla \tilde{g}_{n v_{c}, r}(i-\lfloor n c\rfloor+s)\left(\Pi_{n}(i+1)-\Pi_{n}(i)\right)\right| \\
& \leq\left(\sup _{i^{\prime} \in \mathbb{Z}}\left|\Pi_{n}\left(i^{\prime}-1\right)-\Pi_{n}\left(i^{\prime}\right)\right|\right) \sum_{i \in \mathbb{Z}}\left|\nabla \tilde{g}_{n v_{c}, r}(i-\lfloor n c\rfloor)\right| \\
& \leq \sup _{i \in \mathbb{Z}}\left|\Pi_{n}(i-1)-\Pi_{n}(i)\right| 2\left(n v_{c}\right)^{-1},
\end{aligned}
$$

where the last line uses part 2 of Lemma 3.2. Part 2 of the lemma now follows from Theorem 2.1.

Bounding a further set of expectations that appear repeatedly in the estimates first needs another, technical lemma.

Lemma 3.4. Let $\mu$ be any probability distribution on $\mathbb{Z}$. Suppose that $s, f$, and $h$ are real functions on $\mathbb{Z}$ such that $\|f\|_{\infty}<\infty,\|\Delta s\|_{\infty}<\infty$, and $\|h\|_{l_{1}}<\infty$, which also satisfy the inequality

$$
|s(i) f(i)| \leq|h(i)|+k, \quad I_{1} \leq i<I_{2},
$$

for some integers $I_{1}<I_{2}$ and some $k<\infty$. Then

$$
\begin{aligned}
\left|\sum_{i=I_{1}}^{I_{2}} \mu_{i} s(i) \nabla f(i)\right| \leq & \|f\|_{\left(I_{1}, I_{2}\right)}\|\Delta s\|_{\left(I_{1}, I_{2}\right)}+\|h\|_{l_{1}} \sup _{I_{1} \leq i<I_{2}}\left|\mu_{i}-\mu_{i+1}\right|+k d_{\mathrm{TV}}\left(\mu, \mu * \delta_{1}\right) \\
& +\left|\mu_{I_{1}} s\left(I_{1}\right) f\left(I_{1}-1\right)\right|+\left|\mu_{I_{2}} s\left(I_{2}\right) f\left(I_{2}\right)\right|,
\end{aligned}
$$

where $\|g\|_{\left(I_{1}, I_{2}\right)}:=\sup _{I_{1} \leq i<I_{2}}|g(i)|$.

Proof. It is immediate that

$$
\begin{aligned}
\mid \sum_{i=I_{1}}^{I_{2}} & \mu_{i} s(i) \nabla f(i) \mid \\
\leq & \left|\sum_{i=I_{1}}^{I_{2}-1}\left\{\mu_{i+1} s(i+1)-\mu_{i} s(i)\right\} f(i)\right|+\left|\mu_{I_{1}} s\left(I_{1}\right) f\left(I_{1}-1\right)\right|+\left|\mu_{I_{2}} s\left(I_{2}\right) f\left(I_{2}\right)\right| \\
\leq & \left|\sum_{i=I_{1}}^{I_{2}-1}\left\{\mu_{i+1}-\mu_{i}\right\} s(i) f(i)\right|+\left|\sum_{i=I_{1}}^{I_{2}-1} \mu_{i+1}\{s(i+1)-s(i)\} f(i)\right| \\
& +\left|\mu_{I_{1}} s\left(I_{1}\right) f\left(I_{1}-1\right)\right|+\left|\mu_{I_{2}} s\left(I_{2}\right) f\left(I_{2}\right)\right| .
\end{aligned}
$$

Clearly, the second term is bounded by $\|f\|_{\left(I_{1}, I_{2}\right)}\|\Delta s\|_{\left(I_{1}, I_{2}\right)}$. For the first term, in view of (3.15), we have at most

$$
\sum_{i=I_{1}}^{I_{2}-1}\left\{\left|\mu_{i+1}-\mu_{i}\right||h(i)|\right\}+k \sum_{i=I_{1}}^{I_{2}-1}\left|\mu_{i+1}-\mu_{i}\right|,
$$

which is easily bounded by $\|h\|_{l_{1}} \sup _{I_{1} \leq i<I_{2}}\left|\mu_{i}-\mu_{i+1}\right|+k d_{\mathrm{TV}}\left(\mu, \mu * \delta_{1}\right)$, in view of (2.1). 
Note that the argument also goes through for $I_{1}=-\infty$ and $I_{2}=\infty$, in which case the final two elements in the bound disappear.

Lemma 3.4 is combined with parts 4 and 5 of Lemma 3.2 to give the next corollary, which is used as an ingredient in many of the estimates to be made.

Corollary 3.1. Suppose that $|s(i)| \leq|i-\lfloor n c\rfloor|$ for all $|i| \leq n \delta$. Then, for any $0<\delta^{\prime} \leq \delta$ and all $l \in \mathbb{Z}$ such that $|l| \leq n\left(\delta-\delta^{\prime}\right)$, we have

1. $\left|\mathrm{E}\left\{s\left(Y_{n}+l\right) \nabla \tilde{g}_{n v_{c}, r}\left(Y_{n}+l\right) \mathbf{1}\left[\left|Y_{n}\right| \leq n \delta^{\prime}\right]\right\}\right|$

$$
\leq \frac{1}{n v_{c}} \sup _{|i| \leq n \delta}|\Delta s(i)|+\frac{3 C_{2.1}^{\star}}{n} \sqrt{\log n}+\frac{C_{A .2}}{2 n \sqrt{v_{c}}}+\frac{2\left(C_{\{A .1,1\}}+C_{\{A .1,2\}} / \delta^{\prime}\right)}{n v_{c}} ;
$$

2. $\left|\mathrm{E}\left\{s\left(Y_{n}+l\right) \nabla^{2} \tilde{g}_{n v_{c}, r}\left(Y_{n}+l\right) \mathbf{1}\left[\left|Y_{n}\right| \leq n \delta^{\prime}\right]\right\}\right|$

$$
\leq \frac{2}{n v_{c}} \sup _{|i| \leq n \delta}|\Delta s(i)|+\frac{6 C_{2.1}^{\star}}{n} \sqrt{\log n}+\frac{C_{A .2}}{n^{3 / 2} v_{c}}+\frac{4\left(C_{\{A .1,1\}}+C_{\{A .1,2\}} / \delta^{\prime}\right)}{n v_{c}} .
$$

Proof. We take $\Pi_{n} * \delta_{-l}$ for $\mu$ and either $\tilde{g}_{n v_{c}, r}$ or $\nabla \tilde{g}_{n v_{c}, r}$ for $f$ in Lemma 3.4, noting that parts 4 and 5 of Lemma 3.2 give the appropriate counterparts of (3.15). The first three elements appearing in the bound given by Lemma 3.4 are in turn bounded by using part 1 of Lemma 3.2, Theorem 2.1, and Theorem A.2. The last two are bounded by part 1 of Lemma 3.2 and Theorem A.1.

Proof of Theorem 1.1. We are now in a position to undertake the proof of Theorem 1.1, for which we need to bound the terms $R_{1 n}, R_{2 n}$, and $R_{3 n}$ in (3.2) to order $O\left(n^{-(\alpha+1) / 2} \sqrt{\log n}\right)$. First, we show that $R_{3 n}$ is as small as $O\left(n^{-3 / 2}\right)$. This is because, from Barbour and Jensen (1989, remark to Lemma 2.1), if $X \sim \operatorname{Po}(\mu)$ then

$$
\sup _{k \in \mathbb{Z}} \mathrm{P}(X=k) \leq \frac{1}{2 \sqrt{\mu}} .
$$

Hence, and from Corollary A.1, it easily follows that

$$
R_{3 n}=\sup _{k \in \mathbb{Z}} \widehat{\operatorname{Po}}\left(n v_{c}\right)\{k\} \mathrm{P}\left[Y_{n}<-\left\lfloor n v_{c}\right\rfloor\right]=O\left(\frac{1}{n \sqrt{n}}\right) .
$$

For the quantity $R_{2 n}$ in (3.2), we just use part 2 of Lemma 3.3 to give

$$
R_{2 n}:=n v_{c} \sup _{r \in \mathbb{Z}}\left|\mathrm{E}\left\{\nabla^{2} \tilde{g}_{n v_{c}, r}\left(Y_{n}+1\right)\right\}\right| \leq 2 C_{2.1}^{\star} n^{-1} \sqrt{\log n}
$$

It thus remains to bound $R_{1 n}$. To do so, we consider in turn the expectations of the quantities appearing in (3.3) and in (3.6)-(3.12).

Beginning with the elements of $\mathrm{E}\left\{R\left(n, r ; Y_{n}\right)\right\}$, we first have

$$
\mathrm{E}\left\{\frac{n}{2}\left[\sigma^{2}\left(\frac{Y_{n}+\lfloor n c\rfloor}{n}\right)-\sigma^{2}(c)\right] \nabla \tilde{g}_{n v_{c}, r}\left(Y_{n}\right)\right\},
$$

which is of the form considered in part 1 of Corollary 3.1, with $l=0$ and

$$
s(i):=\frac{n}{2}\left[\sigma^{2}\left(\frac{i+\lfloor n c\rfloor}{n}\right)-\sigma^{2}(c)\right] .
$$


For $|i| \leq n \delta / 2$ and $n \geq 2 / \delta$, we have

$$
|s(i)| \leq \frac{1}{2}\left|i-\left\langle n v_{c}\right\rangle\right|\left\|\left(\sigma^{2}\right)^{\prime}\right\|_{\delta} \quad \text { and } \quad|s(i)-s(i-1)| \leq \frac{1}{2}\left\|\left(\sigma^{2}\right)^{\prime}\right\|_{\delta},
$$

whereas, for $|i|>n \delta / 2$, we have the simple bound

$$
|s(i)| \leq \frac{n}{2}\left[\sigma^{2}(c)+\sum_{j \in \mathbb{Z}} j^{2} c_{j}\left(1+n^{-1}|i|\right)\right],
$$

using assumption (A2). By Theorem A.1 and Corollary A.1, it follows that the latter element contributes at most $O\left(n^{-1}\right)$ to $\left|\mathrm{E}\left\{R\left(n, r ; Y_{n}\right)\right\}\right|$; for the former, Corollary 3.1 gives a bound of order $O\left(n^{-1} \sqrt{\log n}\right)$.

For the next term,

$$
\mathrm{E}\left\{n\left[F\left(\frac{Y_{n}+\lfloor n c\rfloor}{n}\right)-F(c)-\frac{Y_{n}}{n} F^{\prime}(c)\right] \tilde{g}_{n v_{c}, r}\left(Y_{n}\right)\right\},
$$

$\left|\tilde{g}_{n v_{c}, r}\left(Y_{n}\right)\right|$ is bounded by $1 /\left(n v_{c}\right)$, using part 1 of Lemma 3.2. The contribution from the part $\left|Y_{n}\right| \leq n \delta$ is thus easily bounded by

$$
\frac{1}{v_{c}}\left[\left\|F^{\prime \prime}\right\|_{\delta} n^{-2} \mathrm{E}\left\{Y_{n}^{2} \mathbf{1}\left[\mid Y_{n} \leq n \delta\right]\right\}+\left\|F^{\prime}\right\|_{\delta} n^{-1}\right],
$$

and $\mathrm{E}\left\{Y_{n}^{2} \mathbf{1}\left[\mid Y_{n} \leq n \delta\right]\right\}=O(n)$ by Theorem A.1, so that the whole contribution is of order $O\left(n^{-1}\right)$. If $\left|Y_{n}\right|>n \delta$, assumption (A2) and Theorem A.1 guarantee a contribution of the same order. The third term immediately yields

$$
\mathrm{E}\left|F^{\prime}(c)\left\langle n v_{c}\right\rangle \tilde{g}_{n v_{c}, r}\left(Y_{n}\right)\right| \leq \frac{\left|F^{\prime}(c)\right|}{n v_{c}},
$$

again of order $O\left(n^{-1}\right)$. All of these elements are of order $O\left(n^{-1} \sqrt{\log n}\right)$, at least as small as the order $O\left(n^{-(1+\alpha) / 2} \sqrt{\log n}\right)$ stated in the theorem, and it thus remains to bound

$$
\left|\mathrm{E}\left\{E_{n l}\left(\tilde{g}_{n v_{c}, r}, Y_{n}+\lfloor n c\rfloor\right)\right\}\right| \quad \text { for } 1 \leq l \leq 7 .
$$

For the term arising from (3.6), we have

$$
\mathrm{E}\left\{\frac{n}{2}\left[F\left(\frac{Y_{n}+\lfloor n c\rfloor}{n}\right)-F(c)\right] \nabla \tilde{g}_{n v_{c}, r}\left(Y_{n}\right)\right\},
$$

which is of the form considered in part 1 of Corollary 3.1, with $l=0$ and

$$
s(i):=\frac{n}{2}\left[F\left(\frac{i+\lfloor n c\rfloor}{n}\right)-F(c)\right],
$$

and can be treated very much as (3.16) was, yielding a bound of the same order. For the term arising from (3.7),

$$
\mathrm{E}\left\{\sum_{j=2}^{\lfloor\sqrt{n}\rfloor}\left[\sum_{k=2}^{j}\left(\begin{array}{l}
k \\
2
\end{array}\right) \nabla^{2} \tilde{g}_{n v_{c}, r}\left(Y_{n}+j-k+1\right)\right] n \lambda_{j}(c)\right\},
$$


we can use part 2 of Lemma 3.3 to bound the expectations $\mathrm{E}\left\{\nabla^{2} \tilde{g}_{n v_{c}, r}\left(Y_{n}+j-k+1\right)\right\}$, giving a contribution of at most

$$
\sum_{j=2}^{\lfloor\sqrt{n}\rfloor} \frac{1}{6} j^{3} c_{j} n \frac{2 C_{2.1}^{\star}}{n^{2} v_{c}} \sqrt{\log n} \leq \frac{C_{2.1}^{\star} s_{\alpha}}{3 v_{c}} n^{-(1+\alpha) / 2} \sqrt{\log n},
$$

where we have also used assumption (A2).

The next term is from (3.8), and is more complicated. For its summands, we write

$$
\begin{aligned}
{\left[-\left(\begin{array}{l}
j \\
2
\end{array}\right)\right.} & \left.\nabla \tilde{g}_{n v_{c}, r}\left(Y_{n}\right)+\sum_{k=1}^{j-1} k \nabla \tilde{g}_{n v_{c}, r}\left(Y_{n}+j-k\right)\right] n\left\{\lambda_{j}\left(\frac{Y_{n}+\lfloor n c\rfloor}{n}\right)-\lambda_{j}(c)\right\} \\
= & -\left(\begin{array}{l}
j \\
2
\end{array}\right) \nabla \tilde{g}_{n v_{c}, r}\left(Y_{n}\right) n\left\{\lambda_{j}\left(\frac{Y_{n}+\lfloor n c\rfloor}{n}\right)-\lambda_{j}(c)\right\} \\
& +\sum_{k=1}^{j-1} k \nabla \tilde{g}_{n v_{c}, r}\left(Y_{n}+j-k\right) n\left\{\lambda_{j}\left(\frac{Y_{n}+\lfloor n c\rfloor+j-k}{n}\right)-\lambda_{j}(c)\right\} \\
& +\sum_{k=1}^{j-1} k \nabla \tilde{g}_{n v_{c}, r}\left(Y_{n}+j-k\right) n\left\{\lambda_{j}\left(\frac{Y_{n}+\lfloor n c\rfloor}{n}\right)-\lambda_{j}\left(\frac{Y_{n}+\lfloor n c\rfloor+j-k}{n}\right)\right\} \\
= & : E_{n 3}^{(1)}\left(Y_{n}, j\right)+E_{n 3}^{(2)}\left(Y_{n}, j\right)+E_{n 3}^{(3)}\left(Y_{n}, j\right),
\end{aligned}
$$

say. The term $E_{n 3}^{(1)}\left(Y_{n}, j\right)$ is of the form considered in part 1 of Corollary 3.1, with $l=0$ and

$$
s(i):=-n\left(\begin{array}{l}
j \\
2
\end{array}\right)\left\{\lambda_{j}\left(\frac{i+\lfloor n c\rfloor}{n}\right)-\lambda_{j}(c)\right\} .
$$

For $|i| \leq n \delta / 2$,

$$
|s(i)| \leq\left(\begin{array}{l}
j \\
2
\end{array}\right)\left|i-\left\langle n v_{c}\right\rangle\right|\left\|\lambda_{j}^{\prime}\right\|_{\delta} \quad \text { and } \quad|s(i)-s(i-1)| \leq\left(\begin{array}{l}
j \\
2
\end{array}\right)\left\|\lambda_{j}^{\prime}\right\|_{\delta},
$$

whereas, for $|i|>n \delta / 2$, we have the direct bound

$$
|s(i)| \leq n c_{j}\left(\begin{array}{l}
j \\
2
\end{array}\right)\left(2+n^{-1}|i|\right),
$$

using assumption (A2). From Corollary 3.1 and assumption (A4), the contribution from the first part is of order

$$
O\left(c_{j}\left(\begin{array}{l}
j \\
2
\end{array}\right) n^{-1} \sqrt{\log n}\right)
$$

the second part is also at most of this order, in view of Theorem A.1, Corollary A.1, and part 2 of Lemma 3.2. Adding over $j \leq\lfloor\sqrt{n}\rfloor$, this gives a total contribution to the quantity $\left|\mathrm{E}\left\{E_{n 3}\left(\tilde{g}_{n v_{c}, r}, Y_{n}+\lfloor n c\rfloor\right)\right\}\right|$ of order $O\left(n^{-1} \sqrt{\log n}\right)$.

For $E_{n 3}^{(2)}\left(Y_{n}, j\right)$, we now have a sum of terms of the form considered in part 1 of Corollary 3.1, with $l=j-k$ and

$$
s(i):=n k\left\{\lambda_{j}\left(\frac{i+\lfloor n c\rfloor}{n}\right)-\lambda_{j}(c)\right\} .
$$


Supposing $n$ to be large enough that $\sqrt{n} \leq n \delta / 2$, we have

$$
|s(i)| \leq k\left\|\lambda_{j}^{\prime}\right\|_{\delta}\left|i-\left\langle n v_{c}\right\rangle\right| \quad \text { and } \quad|s(i)-s(i-1)| \leq k\left\|\lambda_{j}^{\prime}\right\|_{\delta}
$$

for $|i| \leq n \delta / 2$, whereas, for $|i|>n \delta / 2$, we have the bound

$$
|s(i)| \leq n k c_{j}\left(2+n^{-1}|i|\right) .
$$

Arguing very much as for (3.17), it thus follows that the total contribution to the quantity $\left|\mathrm{E}\left\{E_{n 3}\left(\tilde{g}_{n v_{c}, r}, Y_{n}+\lfloor n c\rfloor\right)\right\}\right|$ is again of order $O\left(n^{-1} \sqrt{\log n}\right)$.

Finally, for $E_{n 3}^{(2)}\left(Y_{n}, j\right)$, we again have a sum of terms. We first note that

$$
\left|\lambda_{j}\left(\frac{i+\lfloor n c\rfloor}{n}\right)-\lambda_{j}\left(\frac{i+\lfloor n c\rfloor+j-k}{n}\right)\right| \leq n^{-1}|j-k|\left\|\lambda_{j}^{\prime}\right\|_{\delta}
$$

for $|i| \leq n \delta / 2$, and this leads to a contribution to $\left|\mathrm{E}\left\{E_{n 3}^{(2)}\left(Y_{n}, j\right)\right\}\right|$ of at most

$$
\sum_{k=1}^{j-1} \frac{k(j-k)\left\|\lambda_{j}^{\prime}\right\|_{\delta}}{n v_{c}} \leq \frac{L_{1} j^{3} c_{j}}{6 n v_{c}}
$$

in view of part 1 of Lemma 3.2. For $|i|>n \delta / 2$, there is the bound

$$
\left|E_{n 3}^{(2)}(i, j)\right| \leq \sum_{k=1}^{j-1} \frac{k}{v_{c}} c_{j}\left\{2+n^{-1}(2|i|+j-k)\right\},
$$

giving

$$
\left|\mathrm{E}\left\{E_{n 3}^{(2)}\left(Y_{n}, j\right) \mathbf{1}\left[\left|Y_{n}\right|>\frac{n \delta}{2}\right]\right\}\right| \leq j^{2} c_{j}\left\{\frac{7}{6} \mathrm{P}\left[\left|Y_{n}\right|>\frac{n \delta}{2}\right]+n^{-1} \mathrm{E}\left\{\left|Y_{n}\right| \mathbf{1}\left[\left|Y_{n}\right|>\frac{n \delta}{2}\right]\right\}\right\},
$$

because $j \leq\lfloor\sqrt{n}\rfloor$. Adding (3.18) and (3.19) over $j \leq\lfloor\sqrt{n}\rfloor$ gives a total contribution to $\left|\mathrm{E}\left\{E_{n 3}^{(2)}\left(Y_{n}, j\right)\right\}\right|$ of order $O\left(n^{-(1+\alpha) / 2}\right)$, because of assumption (A2).

The term from (3.9) is much easier. For $|i| \leq n \delta$, we have the bound

$$
\lambda_{j}\left(\frac{i+\lfloor n c\rfloor}{n}\right) \leq c_{j}(1+\delta)
$$

by assumption (A2), and $\mathrm{E}\left|\nabla \tilde{g}_{n v_{c}, r}\left(Y_{n}+l\right)\right| \leq 2\left(C_{A .2} / v_{c}\right) n^{-3 / 2}$ for any $l$, by Lemma 3.3 . Hence,

$$
\begin{aligned}
\mathrm{E}\left|\left[-\left(\begin{array}{l}
j \\
2
\end{array}\right) \nabla \tilde{g}_{n v_{c}, r}\left(Y_{n}\right)+\sum_{k=1}^{j-1} k \nabla \tilde{g}_{n v_{c}, r}\left(Y_{n}+j-k\right)\right] n \lambda_{j}\left(\frac{Y_{n}+\lfloor n c\rfloor}{n}\right) \mathbf{1}\left[\left|Y_{n}\right| \leq n \delta\right]\right| \\
\quad \leq j^{2} c_{j}(1+\delta) \frac{2 C_{A .2}}{v_{c}} n^{-1 / 2}
\end{aligned}
$$

and summing over $j \geq\lceil\sqrt{n}\rceil$ gives a total contribution to (3.9) of at most

$$
s_{\alpha} \frac{2 C_{A .2}}{v_{c}}(1+\delta) n^{-(1+\alpha) / 2},
$$


in view of assumption (A2). For $\left|Y_{n}\right|>n \delta$, the $j$-contribution is bounded by

$$
j^{2} c_{j}\left\|\nabla \tilde{g}_{n v_{c}, r}\right\|_{\infty} \mathrm{E}\left\{\left(n+\left|Y_{n}\right|\right) \mathbf{1}\left[\left|Y_{n}\right| \geq n \delta\right]\right\} \leq \frac{2 j^{2} c_{j}\left(C_{\{A .1,1\}}+C_{\{A .1,2\}}\right)}{n v_{c}},
$$

in view of part 1 of Lemma 3.2 and Theorem A.1, and summing over $j \geq\lceil\sqrt{n}\rceil$ gives a contribution of order $O\left(n^{-1-\alpha / 2}\right)$. Hence, the complete contribution from (3.9) is of order $O\left(n^{-(1+\alpha) / 2}\right)$.

The remaining terms (3.10)-(3.12) are treated in exactly the same way as those in (3.7)-(3.9). In all, the largest order of any of the terms in (3.6)-(3.12) is $O\left(n^{-(1+\alpha) / 2} \sqrt{\log n}\right)$, and since the other terms were of order $O\left(n^{-1} \sqrt{\log n}\right)$, Theorem 1.1 is proved.

\section{Appendix A}

The following results from Socoll and Barbour (2009) are used in the proofs.

Theorem A.1. (Socoll and Barbour (2009, Theorem 2.1).) Under assumptions (A1)-(A4), for all large enough $n$, the process $Z_{n}$ has an equilibrium distribution $\Pi_{n}$, and

$$
\begin{gathered}
\mathrm{E}\left\{\left|n^{-1} Z_{n}-c\right| \mathbf{1}\left[\left|n^{-1} Z_{n}-c\right|>\delta\right]\right\} \leq C_{\{A .1,1\}} n^{-1}, \\
\mathrm{E}\left\{\left(n^{-1} Z_{n}-c\right)^{2} \mathbf{1}\left[\left|n^{-1} Z_{n}-c\right| \leq \delta\right]\right\} \leq C_{\{A .1,2\}} n^{-1},
\end{gathered}
$$

for $\delta$ as in assumption (A3) and constants $C_{\{A .1,1\}}$ and $C_{\{A .1,2\}}$; as before, in such expressions, $Z_{n}$ is used to denote a random variable having equilibrium distribution $\Pi_{n}$.

Corollary A.1. (Socoll and Barbour (2009, Corollary 2.5).) Under assumptions (A1)-(A4), for any fixed $\delta^{\prime}$ such that $0<\delta^{\prime} \leq \delta$, there exists $C_{A .1}\left(\delta^{\prime}\right)<\infty$ such that

$$
\mathrm{P}\left[\left|n^{-1} Z_{n}-c\right|>\delta^{\prime}\right] \leq C_{A .1}\left(\delta^{\prime}\right) n^{-1} \text {. }
$$

Lemma A.1. (Socoll and Barbour (2009, Lemma 3.1).) Under assumptions (A1)-(A4), for any $U>0$ and $0<\eta \leq \delta$, there exists a constant $K_{U, \eta}<\infty$ such that

$$
\mathrm{P}\left[\sup _{t \in[0, U]}\left|Z_{n}(t)-n c\right|>n \eta \mid Z_{n}(0)=i\right] \leq n^{-1} K_{U, \eta},
$$

uniformly in $|i-n c| \leq(n \eta / 2) \exp \left\{-\left\|F^{\prime}\right\|_{\delta} U\right\}$.

Theorem A.2. (Socoll and Barbour (2009, Theorem 3.2).) Under assumptions (A1)-(A4), there exists a constant $C_{A .2}>0$ such that

$$
d_{\mathrm{TV}}\left\{\Pi_{n}, \Pi_{n} * \delta_{1}\right\} \leq C_{A .2} n^{-1 / 2},
$$

where $\Pi_{n} * \delta_{1}$ denotes the unit translate of $\Pi_{n}$.

Finally, we shall use the following result, which was used in Socoll and Barbour (2009) to prove the previous theorem; see, for example, Equation (3.7) in the proof of Socoll and Barbour (2009, Theorem 3.2).

Lemma A.2. Under assumptions (A1)-(A4), for any $U \geq 1$, there exists a constant $K_{U}<\infty$ such that

$$
d_{\mathrm{TV}}\left\{\mathcal{L}\left(Z_{n}(U) \mid Z_{n}(0)=i\right), \mathcal{L}\left(Z_{n}(U) \mid Z_{n}(0)=i\right) * \delta_{1}\right\} \leq K_{U} n^{-1 / 2},
$$

uniformly in $|i-n c| \leq n \delta / 2$. 


\section{References}

BARBOUR, A. D. (2008). Coupling a branching process to an infinite dimensional epidemic process. To appear in ESAIM Prob. Statist.

Barbour, A. D. and Jensen, J. L. (1989). Local and tail approximations near the Poisson limit. Scand. J. Statist. 16, 75-87.

Barbour, A. D., Holst, L. And Janson, S. (1992). Poisson Approximation. Oxford University Press.

Chen, L. H. Y. (1975). Poisson approximation for dependent trials. Ann. Prob. 3, 534-545.

Chung, F. And Lu, L. (2006). Concentration inequalities and martingale inequalities: a survey. Internet Math. 3, 79-127.

Hamza, K. and Klebaner, F. C. (1995). Conditions for integrability of Markov chains. J. Appl. Prob. 32, 541-547.

Kurtz, T. G. (1981). Approximation of Population Processes (CBMS-NSF Regional Conf. Ser. Appl. Math. 36). SIAM, Philadelphia, PA.

Röllin, A. (2005). Approximation of sums of conditionally independent random variables by the translated Poisson distribution. Bernoulli 11, 1115-1128.

Socoll, S. And Barbour, A. D. (2009). Translated Poisson approximation to equilibrium distributions of Markov population processes. To appear in Methodology Comput. Appl. Prob. 